\title{
Téoros
}

Revue de recherche en tourisme

\section{Émergence des nouvelles formules en hôtellerie}

\section{Gilles Larivière et Jocelyn Jussaume}

Volume 23, numéro 3, automne 2004

Regards sur l'hôtellerie : un secteur en mutation

URI : https://id.erudit.org/iderudit/1071226ar

DOI : https://doi.org/10.7202/1071226ar

Aller au sommaire du numéro

Éditeur(s)

Université du Québec à Montréal

ISSN

0712-8657 (imprimé)

1923-2705 (numérique)

Découvrir la revue

Citer cet article

Larivière, G. \& Jussaume, J. (2004). Émergence des nouvelles formules en hôtellerie. Téoros, 23(3), 10-16. https://doi.org/10.7202/1071226ar d'utilisation que vous pouvez consulter en ligne.

https://apropos.erudit.org/fr/usagers/politique-dutilisation/ 


\section{Émergence des nouvelles formules en hôtellerie}

\section{Cilles Larivière et Jocelyn Jussaume}

Bien que riche en rebondissements, l'histoire moderne du secteur hôtelier en Amérique du Nord est difficile à découper en séquences précises, alors qu'elle fut modelée au fil d'événements divers qui semblent souvent n'avoir aucun lien entre eux. En accéléré ou au ralenti, tantôt à cause de changements technologiques, sociaux, ou encore en raison du génie créatif ou même simplement par opportunisme, ce secteur s'est forgé, laissant derrière lui plusieurs balises qui nous permettent tout de même de mieux comprendre son évolution.

\section{Les tournants historiques de I'hôtellerie}

À prime abord, les innovations les plus marquantes constatées au sein du secteur hôtelier, au cours des cinq dernières décennies principalement, peuvent apparaître comme de grandes nouveautés. Mais il faut toutefois se rappeler que ces innovations n'ont été, en fait, que le fruit d'ajustements en réponse à d'autres changements sociaux et à d'autres avancées technologiques survenues au cours de l'histoire, plus précisément depuis les cinquante dernières années.

Bien sûr, il faut reconnaître que l'hôtellerie, telle que nous la connaissons aujourd'hui, a constamment évolué et que ces changements se sont toujours faits en réaction à des tournants d'histoire qui apportaient des modifications majeures au mode de vie des populations, notamment au transport des personnes et aux télécommunications. Ainsi, le tableau 1 permet d'affirmer, entre autres, que l'évolution des moyens de transport a directement marqué l'évolution du produit hôtelier. À titre d'exemple, l'avènement des hôtels « aéroport » et « à congrès » n'est-il pas survenu au cours des années 1950 et 1960, peu de temps après le début des vols d'avions à réaction commerciaux? Ces nouveaux types d'hôtels répondaient donc à un besoin émergent.

Bien que le nombre d'unités d'hébergement commercial ait été multiplié par dix depuis les cent dernières années, environ la moitié de ces unités ont été construites au cours des 30 à 35 dernières années, période durant laquelle les moyens de transport se sont rapidement multipliés et améliorés, et durant laquelle les coûts de déplacement sont devenus de plus en plus abordables et accessibles.

Le secteur hôtelier a connu ses plus importantes modifications, au concept même, à la fin des années 1960. Jusque-là, il existait essentiellement deux types d'établissements: les hôtels de grandes villes, qui ont véritablement fait leur apparition au début du dernier siècle, et les motels routiers, formule véritablement popularisée au départ par Holiday Inn et s'adressant au secteur industriel.

À partir de ce moment, le vaste «parc hôtelier» nord-américain (États-Unis et Canada) a connu des vagues successives d'ajouts, mais, de plus en plus, ces nouveaux hôtels étaient destinés à des clien-

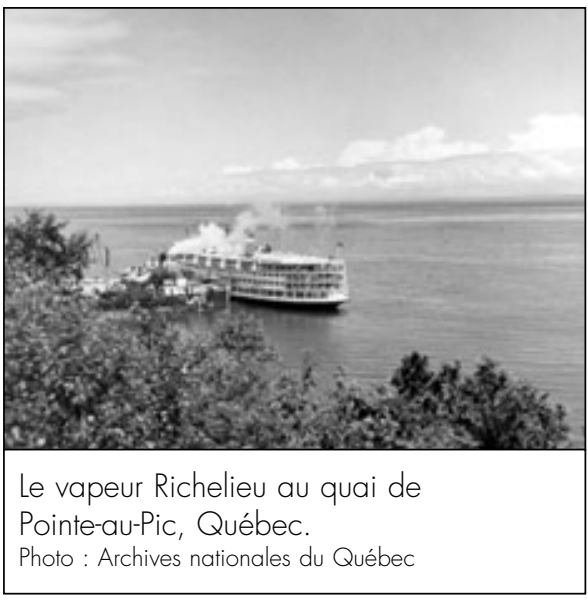

tèles précises, comme les voyageurs en transit aux aéroports, les gens d'affaires, les congressistes, etc. Ensuite, la notion de «segmentation» des produits et des clientèles hôtelières est réellement apparue dans le jargon des professionnels.

La première vague, à la fin des années 1960 et au début des années 1970, a été marquée par la prolifération d'établissements dits «économiques». En soi, ce concept n'était pas nouveau alors que l'on rencontre des motels routiers depuis le milieu des années 1920 aux États-Unis. Cependant, à cette époque, ces établissements faisaient partie de l'hôtellerie «traditionnelle». Alors que plusieurs étaient considérés comme des hôtels « quatre étoiles » lors de leur mise en œuvre, cette perception glissait alors lentement au fil du temps et de l'usure graduelle que connaissaient ces hôtels, pour terminer par la suite au bas de la gamme en chambres «deux étoiles» et, éventuellement, s'éteindre ou demeurer à ce niveau. 
TABLEAU 1 : Faits saillants du secteur hôtelier en Amérique du nord

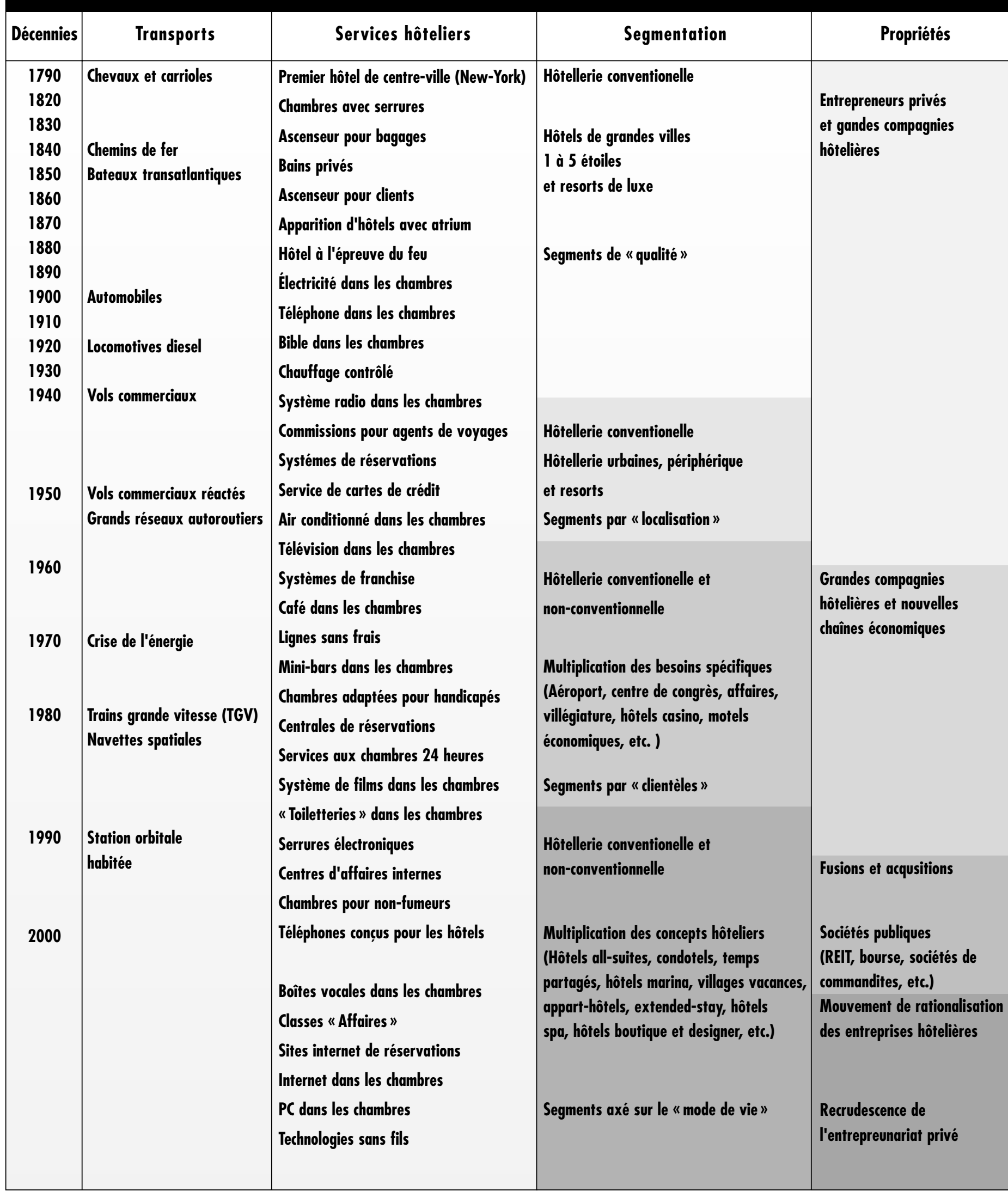




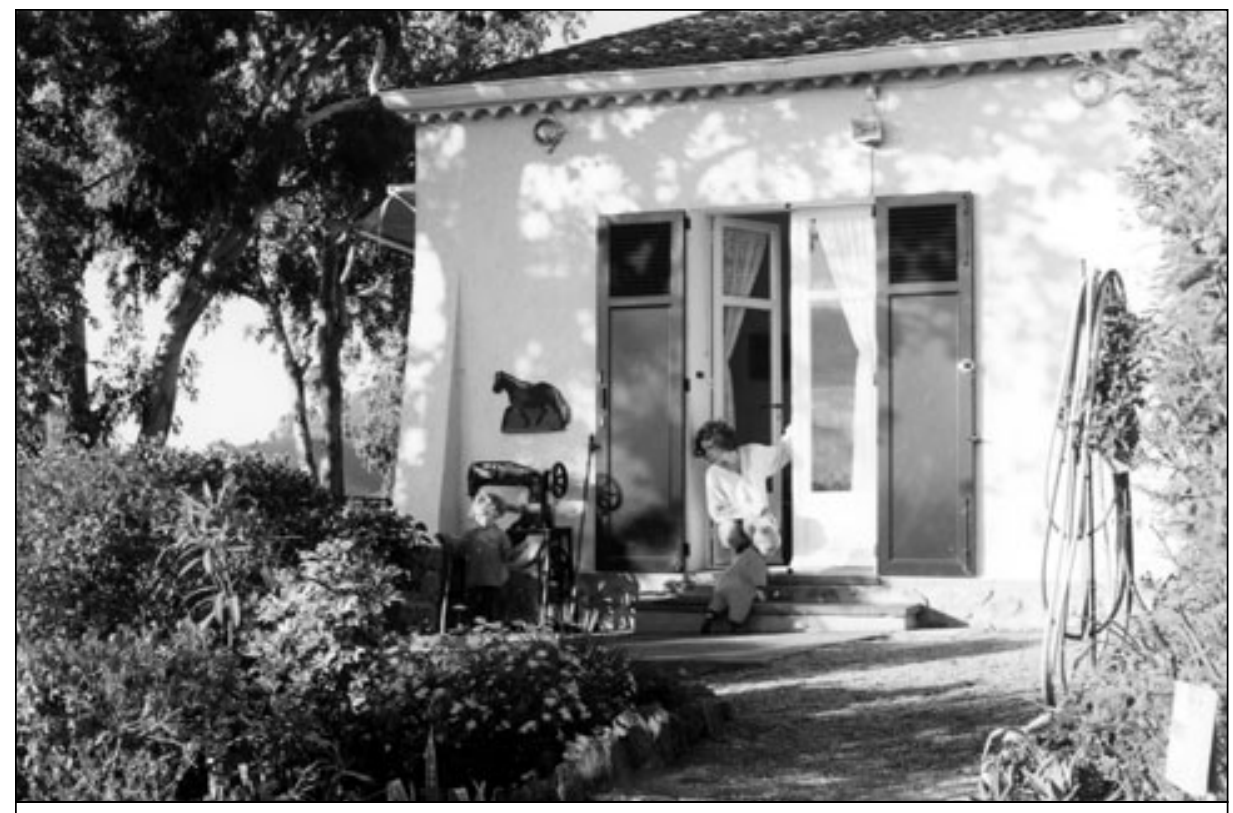

Maisonnette à Théoule-sur-Mer, France photo: Cathy Quessy

La vague des hôtels économiques des années 1970 était différente en raison de la motivation première qui était justement de construire des hôtels qui s'adressaient sciemment à une clientèle économique de moyen et de bas de gamme.

Devant les succès économiques à répétition que connaissait cette formule, de plus en plus d'observateurs se sont alors intéressés à ce secteur et la période 1970-1980 est devenue le cadre de la mise en marché, principalement par les chaînes hôtelières alors en forte montée, de centaines de milliers d'unités de ce genre.

Il faut se rappeler que cette explosion de nouveaux hôtels était alors au cœur de grands changements sociaux en Amérique du Nord. Souvenons-nous qu'à cette période la génération des baby-boomers venait d'entrer de plain-pied sur le marché du travail et que la croissance démographique était soutenue. On assistait alors à une hausse quasi généralisée du niveau de vie des populations urbaines. Du même coup, les moyens de transport permettaient dès lors une grande mobilité des personnes, sur de longues distances et à des coûts accessibles à tous. De plus, cette période coïncidait aussi avec le «droit aux vacances », concept qui auparavant était plutôt considéré comme un privilège.

\section{La segmentation}

Avec l'évolution rapide des habitudes de vie, la notion de «segmentation» a, du même coup, connu elle aussi différents stades à l'intérieur d'une courte période. Alors qu'initialement, avec l'arrivée des hôtels traditionnels durant la première moitié du siècle, on parlait strictement d'une segmentation de produit axé sur la «qualité », généralement évaluée de une à cinq étoiles. Rapidement, on en est venu à faire quelques nuances avec les hôtels urbains, les hôtels routiers et certains établissements situés en villégiature, ces derniers étant souvent considérés comme exclusifs aux classes bien nanties de la société. On pouvait maintenant établir de façon simple une segmentation par type de produit, axée sur la «localisation».

Mais l'importance des changements sociaux, culturels et technologiques survenus au cours des 50 dernières années a engendré des besoins émergents spécifiques qui ont entraîné la construction d'hôtels dédiés, par la force des choses, à des catégories spécifiques de clients. Il était maintenant possible de segmenter non seulement les hôtels, mais aussi les «clientèles ».

Depuis les 15 à 20 dernières années, la concurrence de plus en plus marquée entre les hôteliers a incité ceux-ci à inno- ver davantage. Les méthodes de plus en plus raffinées de mise en marché, conjointement aux moyens grandissants des clientèles, ont poussé les hôteliers à offrir toute une gamme de produits axés sur le mode de vie des clientèles ciblées. Souvenons-nous de certaines publicités récentes qui vantent le cachet home away from home, des produits all-suites ou encore des hôtels-boutiques et designers qui, pour certains, offrent une réplique du style de vie européen ou new-yorkais. Ces courants nous ont ainsi amenés à considérer la segmentation basée sur le «style » ou le mode de vie, et chacun de ces styles était aussi sujet à s'ajuster de nouveau.

À ce sujet, l'exemple de l'arrivée des hôtels all-suites, au début des années 1980, demeure intéressant. À l'origine, ce concept avait été développé pour le marché des femmes d'affaires d'abord. Cependant, les objectifs vis-à-vis de cette clientèle ayant été promptement atteints, cette formule a aussi connu du succès auprès d'autres marchés de consommateurs. Toute une série de concepts dérivés de l'hôtel all-suites, tels les «hôtels-appartements », les hôtels « long-séjour » ou extended stay, les condotels et les formules à temps partagé ont surgi alors presque immédiatement.

Aujourd'hui, en fait, la dénomination «hôtel» semble avoir de moins en moins sa place alors que la multiplication des concepts d'unités et d'établissements tend maintenant plutôt à favoriser l'utilisation du terme "établissement d'hébergement commercial » pour englober l'ensemble de tout ce qui est maintenant disponible. Sur cette base, nous pensons pouvoir affirmer que l'un des plus grands facteurs à avoir marqué le secteur hôtelier au cours des 50 dernières années a sans doute été la forte segmentation des produits et des clientèles. Depuis le début des années 1990, le plus récent phénomène ou concept à marquer ce secteur a été l'arrivée des hôtels «boutiques » et «designers » ou «design » qui, selon nous, identifie peut-être aussi la fin d'un cycle, mais nous y reviendrons ultérieurement. 


\section{Les structures de propriété}

Il faut se rappeler que, parallèlement à l'évolution rapide des produits d'hébergement commercial, le secteur a connu de très importantes modifications quant à la structure de propriété de ces établissements. Avant la fin des années 1960, l'hôtellerie était un commerce détenu et opéré principalement par des entrepreneurs privés, c'est-à-dire des individus et quelques familles, souvent localisé en périphérie des grandes agglomérations, ainsi que par des grands groupes hôteliers du temps comme Westin (au début Western Hotels), Sheraton, Hilton, Marriott ou Holiday Inn, qui détenaient essentiellement les grands hôtels de centres-villes. L'arrivée massive des hôtels économiques et des chaînes hôtelières en périphérie des grands centres a cependant vite fait de changer la donne.

Majoritairement localisés en périphérie des grandes villes, ces nouveaux hôtels avec bannière s'étendaient stratégiquement sur le territoire pour le compte d'entreprises hôtelières fortement structurées. Cela venait drôlement changer la nature de l'hôtellerie à une époque où la majorité des établissements étaient détenus et exploités par des entrepreneurs indépendants. Soulignons le fait que ces ajouts ont été réalisés à un rythme fou, alors que certaines de ces chaines annonçaient même, à grand renfort de publicité, avoir maintenu un rythme d'ouverture de deux établissements par semaine pendant deux ans.

Ainsi, le monde financier a rapidement remarqué cette poussée du secteur hôtelier et surtout les succès économiques que connaissaient un grand nombre de ces établissements ainsi que leurs chaînes affiliées. La forte croissance de la demande enregistrée à cette époque coïncidait aussi avec une période de forte inflation dont ces hôteliers ont su bénéficier. La grande souplesse que procure le produit hôtelier quant à l'ajustement des tarifs, et ce sur une base quotidienne, démarquait alors ce secteur en comparaison avec celui de l'immobilier qui ne pouvait, par exemple, offrir un tel avantage. Plusieurs investisseurs ont alors vu poindre de nouvelles occasions d'affaires et voyaient un fort potentiel dans le secteur de l'hôtellerie.
L'amorce d'une ère de mondialisation favorisant l'accès à de nouveaux marchés internationaux ouvrait, du même coup, toutes grandes les portes pour maintenir la croissance de l'offre hôtelière notamment. Aux yeux de la majorité des investisseurs, cela représentait une double occasion d'investir en ce domaine.

Les performances historiques positives jumelées au nouveau besoin de « réseautage » entre les établissements, en raison de la nécessité de suivre et de desservir le voyageur où qu'il aille, ont suscité, dès lors, la première vague de fusions et d'acquisitions à travers le secteur hôtelier. Cela permettait ainsi une intégration accrue du produit face à un client que l'on voulait fidéliser, peu importe sa destination. À défaut de pouvoir l'héberger au sein de la même chaîne, la fusion permettait que le client demeure à tout le moins dans la même famille hôtelière.

Toutefois, ce nouveau besoin de fusion des entreprises hôtelières a nécessité l'utilisation de nouveaux modes de financement qui ont alors fait leur apparition de façon directe dans ce secteur. Rappelons-nous les appels à l'épargne et les cotations en bourse. Certaines chaînes ont toutefois vu jaillir cette opportunité bien avant les autres. Ainsi, l'entreprise hôtelière Sheraton a été la première à s'inscrire en bourse, dès 1945, au New York Stock Exchange.

Soudainement, le secteur hôtelier, qui avait été depuis toujours majoritairement privé et indépendant, devenait un produit appartenant à des institutions collectives du type REIT (Real Estate Investment Trust), sociétés en commandites ou autres regroupements spécialisés d'épargnes.

Ce faisant, le secteur hôtelier doit, aujourd'hui encore, trouver de nouvelles formules pour stimuler l'intérêt des investisseurs dans un contexte qui n'égale en rien celui du boom hôtelier des années 1970 et 1980, alors que les outils financiers désormais en place sont devenus beaucoup plus techniques et complexes que ceux initiés il y a une vingtaine d'années. Comme pour la plupart des secteurs,

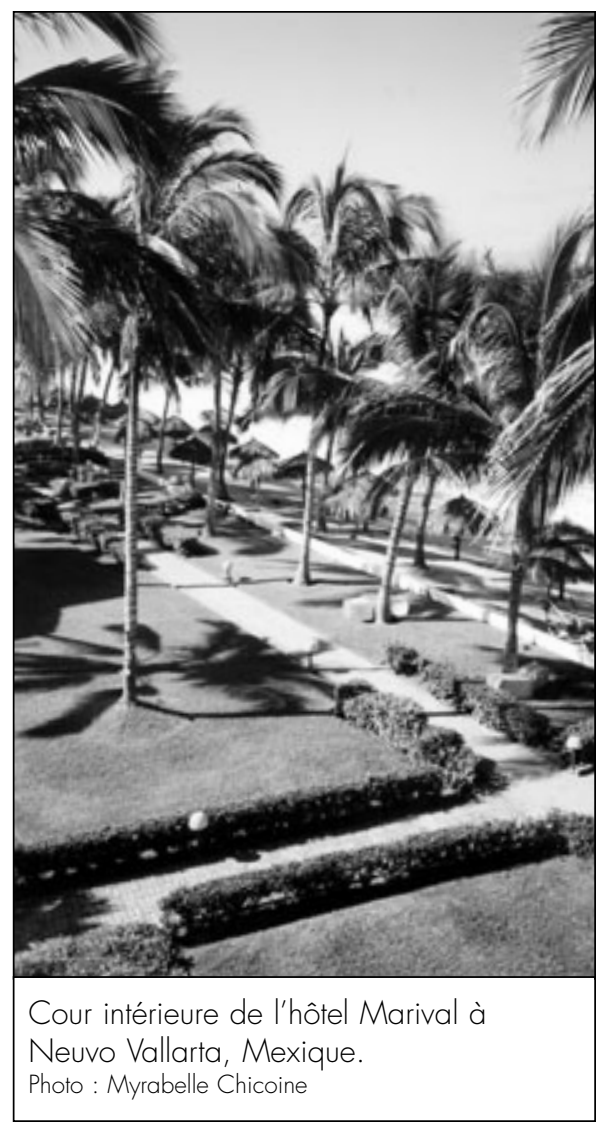

le cycle de ce secteur est fortement influencé par l'équilibre entre l'offre et la demande qui, dans ce cas, est sujet aux variations engendrées par plusieurs facteurs externes imprévisibles comme les tendances économiques, le climat géopolitique, la météo, les courants populaires, les transports, etc. Les observateurs reconnaissent maintenant que l'hôtellerie est un secteur beaucoup plus vulnérable qu'on ne l'envisageait auparavant.

\section{La fin d'un cycle ?}

La période des années 1990 a aussi été le théâtre de nombreux mouvements de fusions et d'acquisitions. Cependant, au cours des cinq dernières années, on constate que cette tendance tend à se résorber alors que ces entreprises entrent maintenant dans une période de consolidation de l'inventaire. Alors que la priorité, jusqu'au début des années 2000, était de détenir le maximum d'unités dans le marché, les entreprises procèdent maintenant à l'épuration de leur parc hôtelier afin de canaliser efficacement leur capacité et leurs efforts. 
Bien que ces entreprises hôtelières continuent tout de même d'offrir une vaste gamme de produits, celles-ci doivent maintenant tenter de limiter le risque lié à l'exploitation de leurs sous-produits moins performants ou plus volatiles, dans le but d'améliorer les rendements à court terme pour le compte des propriétaires, c'est-à-dire les actionnaires qui exigent des résultats immédiats.

La montée graduelle des hôtels-boutiques et designers, au cours des années 1990 et encore aujourd'hui, marque le plus récent courant à survenir au sein du secteur hôtelier. Mais nous sommes de ceux qui croient que, cette fois, le phénomène est plus qu'une simple mode. Bien sûr ces hôtels répondent à la base, comme nous l'avons affirmé précédemment, à des besoins sociaux changeants liés au mode de vie et à la reconnaissance, mais nous pensons qu'ils se sont aussi développés grâce à la réaction de la part de certains segments fortement utilisateurs, face à une hôtellerie de plus en plus standardisée, aseptisée en matière de service et démesurée en taille.

Ayant toujours l'œil aguerrit, les chaînes hôtelières ont vite tenté de profiter de ce courant en mettant sur le marché leurs propres produits très personnalisés au sein même de leurs produits existants. Par exemple, en modifiant quelques étages et en annonçant des formules plus sélectives comme les «formules club », «étages privilèges », etc., comme ce slogan de l'hôtel Fairmont Royal York de Toronto qui présente ainsi ses unités Fairmont Gold: «luxury boutique hotel within a hotel».

Certains entrepreneurs indépendants ont vite compris que le marché était disposé à s'enrichir d'un produit au concept davantage axé sur la dimension humaine. Le temps était venu de créer des hôtels directement adaptés à leurs clientèles, moins élaborés mais mieux localisés, de plus petite taille et aux services et aux ambiances très personnalisés, tant en ce qui concerne l'hébergement que les services de restauration. Il était clair qu'avec un tel produit les clients seraient enclins à payer une prime pour bénéficier de ces avantages et de ces distinctions.

\section{Les hôtels " boutiques "}

Le terme hôtel «boutiques », qui devient de plus en plus populaire, est apparu au cours des années 1980, grâce à l'émergence du travail d'entrepreneurs créateurs, que l'on identifie aujourd'hui comme étant les pionniers du concept, Steve Rubell et Ian Schrager à New York et Bill Kimpton à San Francisco.

MM. Rubell et Schrager ont d'abord établi leur réputation comme co-propriétaires de la célèbre discothèque «Studio 54 » à New York. Aujourd'hui, l'entreprise diversifiée est dirigée par Ian Schrager et est davantage reconnue à cause de ses quatre hôtels prestigieux de New York: le Morgan's, le Royalton, le Paramount et le Hudson. Cette chaîne possède ou gère une dizaine d'établissements aux États-Unis et à Londres, tous aussi exclusifs les uns que les autres. La plupart des hôtels détenus par Ian Schrager mettent de l'avant le fait qu'ils ont été conçus par de célèbres designers tels Philippe Starck et Andrée Putman. Ces hôtels sont devenus en vogue à cause de l'atmosphère, de l'ambiance, du cachet, du décor, des mobiliers et des éclairages absolument hors du commun, créés par ces deux remarquables designers tout à fait innovateurs.

Quant à Bill Kimpton, il a acheté son premier hôtel, le Bedford Hotel, en 1981.
Aujourd'hui, il opère près d'une quarantaine d'hôtels dits de style «européen » aux États-Unis et au Canada. Son entreprise, Kimpton Group, est aussi à la base de la création de la chaîne d'hôtels-boutiques « Hôtels Monaco » qui compte sept établissements.

Le concept de l'hôtel «boutique » est bien ancré dans le secteur hôtelier d'aujourd'hui. Starwood Hotels \& Resorts, l'une des plus grandes compagnies hôtelières au monde, a décidé d'entrer dans ce marché en lançant sa propre chaîne et son propre concept d'hôtel «boutique», sous la bannière «W Hotels ». Starwood possède présentement 20 hôtels «W », dont celui de Montréal qui prévoit ouvrir ses portes dès l'automne 2004. L'intention véhiculée dans chaque hôtel « W» est que chacun d'eux puisse refléter la personnalité de la ville dans laquelle il se trouve, ce qui leur procure un caractère distinct et à la fois contemporain, tout en maintenant les mêmes standards de service et la même qualité sur le plan des installations.

\section{" Boutique " versus " designer "}

Alors que le concept « hôtel-boutique » est devenu de plus en plus populaire aux yeux des développeurs, en tant que produit hôtelier, nous constatons que, avec le temps, cette

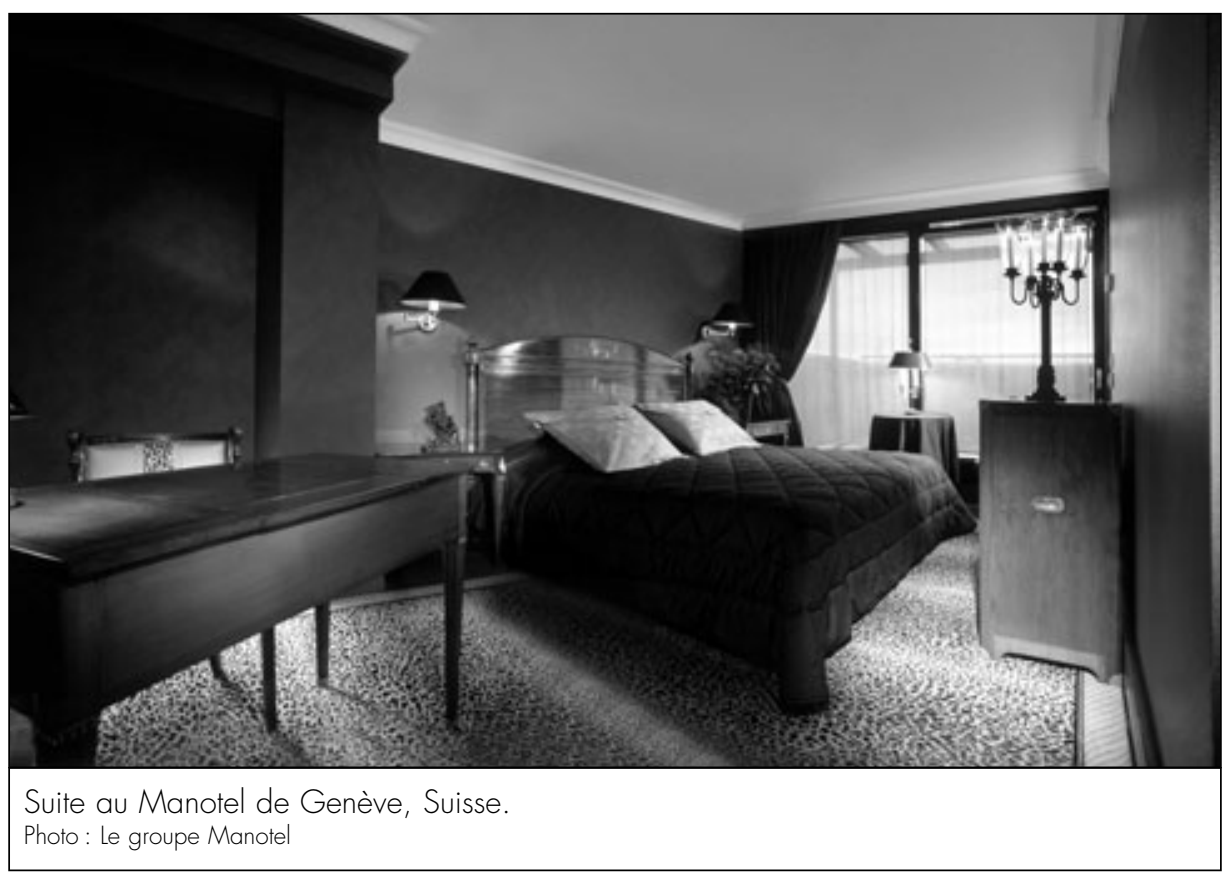


notion a été largement galvaudée depuis et que le terme «boutique » représente un défi d'interprétation à cause du large usage dont il fait aujourd'hui l'objet pour désigner cette nouvelle gamme de produits hôteliers développés au cours des dernières années.

Avec le succès qu'ont connu les précurseurs du concept, ainsi que l'arrivée sur la scène de Starwood et de sa chaîne d'hôtels «W $\mathrm{W}$, nous avons remarqué que le concept de base avait plutôt connu une évolution continue du produit boutique. C'est l'observation et l'analyse de cette évolution qui nous ont permis en fait de définir un autre courant émergent issu du concept original. Nous parlons donc aussi aujourd'hui d'hôtel « designer » ou « design ».

Ces hôtels sont délibérément différents les uns des autres. Ils sont aménagés dans le but d'exprimer l'individualité du propriétaire, projetant même dans bien des cas l'expression d'un état d'âme ou d'un point de vue. À l'origine, la plupart des hôtels-boutiques ont été conçus à partir de vieux hôtels et d'édifices en décrépitude qui avaient une valeur historique ou qui possédaient un certain caractère, et qui, une fois rénovés, afficheraient un style, des couleurs et une personnalité bien distincts. L'atmosphère et l'ambiance font donc de chaque hôtel-boutique un endroit unique. Cet aspect est essentiel.

L'hôtel-boutique, au sens propre, est généralement de petite dimension, offrant en moyenne de 100 à 200 chambres, ce qui contribue dès le départ à créer l'atmosphère intimiste recherchée par le concept. Cette intimité et cette ambiance permettent aux hôtels d'offrir un service attentionné et personnalisé à chacun de leurs clients. Cela a pour effet de générer pour ces établissements un excellent taux de retour de la clientèle, en comparaison avec l'hôtellerie en général.

Entre-temps, nous constatons qu'au fil de leur évolution les hôtels-boutiques ont eu tendance à gagner en style ou en personnalité, transcendant de plus en plus la vision et le caractère du propriétaire, alors que le facteur «taille» était de moins en moins considéré. Ce glissement du concept original a maintenant sa propre identité, soit l'hôtel-designer.
Le Paramount Hotel de New York, hôtel de 601 chambres appartenant à Ian Schrager, est un premier exemple de concept d'hôtel-designer. Ainsi, on peut difficilement associer cet hôtel à un hôtel-boutique en termes de services personnalisés et de dimension ; cependant, le Paramount Hotel présente un style et un design tout à fait uniques et propres au concept d'hôtel-boutique.

Au cours des dernières années, New York a aussi assisté à l'ouverture d'hôtels-designers tels le Soho Grand Hotel et le Mercer Hotel, créations respectivement des concepteurs Bill Sofield et Andre Balazs. À l'instar des hôtels d'Ian Schrager, l'apparence extérieure de ces établissements est un modèle de finesse empreinte de subtilité. Aucune enseigne n'apparaît à l'extérieur de l'immeuble, pour annoncer le nom de l'hôtel ou même qu'il s'agit d'un hôtel.

Au même titre que le haut niveau de service, les hôtels-boutiques et designers se définissent aussi par leurs installations de restauration, disposant de restaurants et de bars dédiés davantage à une clientèle «jet-set» ou encore «branchée ». Ainsi, non seulement la qualité et le style des installations de restauration sont importants dans le développement et le succès d'un produit designer, mais également la signature qu'arborent les restaurants et les bars. Bien que cela doive tout aussi bien s'appliquer aux hôtels-boutiques, il se trouve qu'à ce chapitre l'hôtel-designer se surpassera en s'associant à de grands noms du milieu tels Rande Gerber, Drew Nieporent et Gail DeFerrari.

Alors que les hôtels-boutiques proposent généralement des établissements de petite taille fortement axés sur le service et peuvent afficher des styles variés allant de l'ultra conservateur au très «branché », les hôtels-designers, quant à eux, n'ont pas de critère de taille, conservent en général un niveau de service standard et se caractérisent fortement par l'aspect design, des décors ambitieux ainsi qu'une atmosphère et une ambiance éclatées, parfois même surréalistes.

\section{La revanche des indépendants}

En comparaison avec l'hôtellerie traditionnelle, les hôtels-boutiques sont, la plupart du temps, positionnés sur le marché des produits hôteliers première classe et sont en mesure de commander des tarifs du niveau de ceux affichés par les plus grandes chaînes hôtelières telles Sheraton, Marriott et Hilton. Cela est rendu possible à cause du caractère unique, du service très personnalisé et de la plus value offerts par le produit «boutique ».

Depuis le début de ce courant, cette formule connaît un succès certain, notamment dans les grandes villes des États-Unis et du Canada où la concentration d'offre hôtelière traditionnelle était déjà forte, comme New York, Miami, Los Angeles, San Francisco, Vancouver et, plus récemment, Toronto et Montréal.

De notre point de vue, l'arrivée des hôtelsboutiques et designers est peut-être le signe avant-coureur de la fin d'un cycle pour le secteur hôtelier qui aura duré environ une quarantaine d'années. Alors que le secteur hôtelier de l'époque était dominée par les entrepreneurs indépendants, les entreprises hôtelières de toutes catégories sont alors venues submerger le marché d'innombrables produits tous aussi diversifiés les uns que les autres et ont dicté les règles de ce secteur jusqu'au début des années 2000. Le prix à payer pour le développement de ces entreprises hôtelières aura été le partage des acquis avec le monde financier et les regroupements collectifs. Mais on assiste maintenant à une recrudescence de la participation des entrepreneurs indépendants au sein du courant de l'hôtellerie boutique qui, cette fois, sont en mesure d'offrir une alternative solide aux modèles de l'hôtellerie traditionnelle, un beau retour des choses !

Nous pensons que les hôtels-boutiques sont là pour rester. Ils subiront certes des modifications, leur nombre s'ajustera au fil du temps, certains deviendront même des gîtes touristiques, mais les vraies valeurs véhiculées par la formule persisteront, c'est-à-dire des hôtels de taille humaine, très personnalisés sur le plan des services, mais conservant la touche bien terre à terre de l'hôtellerie traditionnelle. 


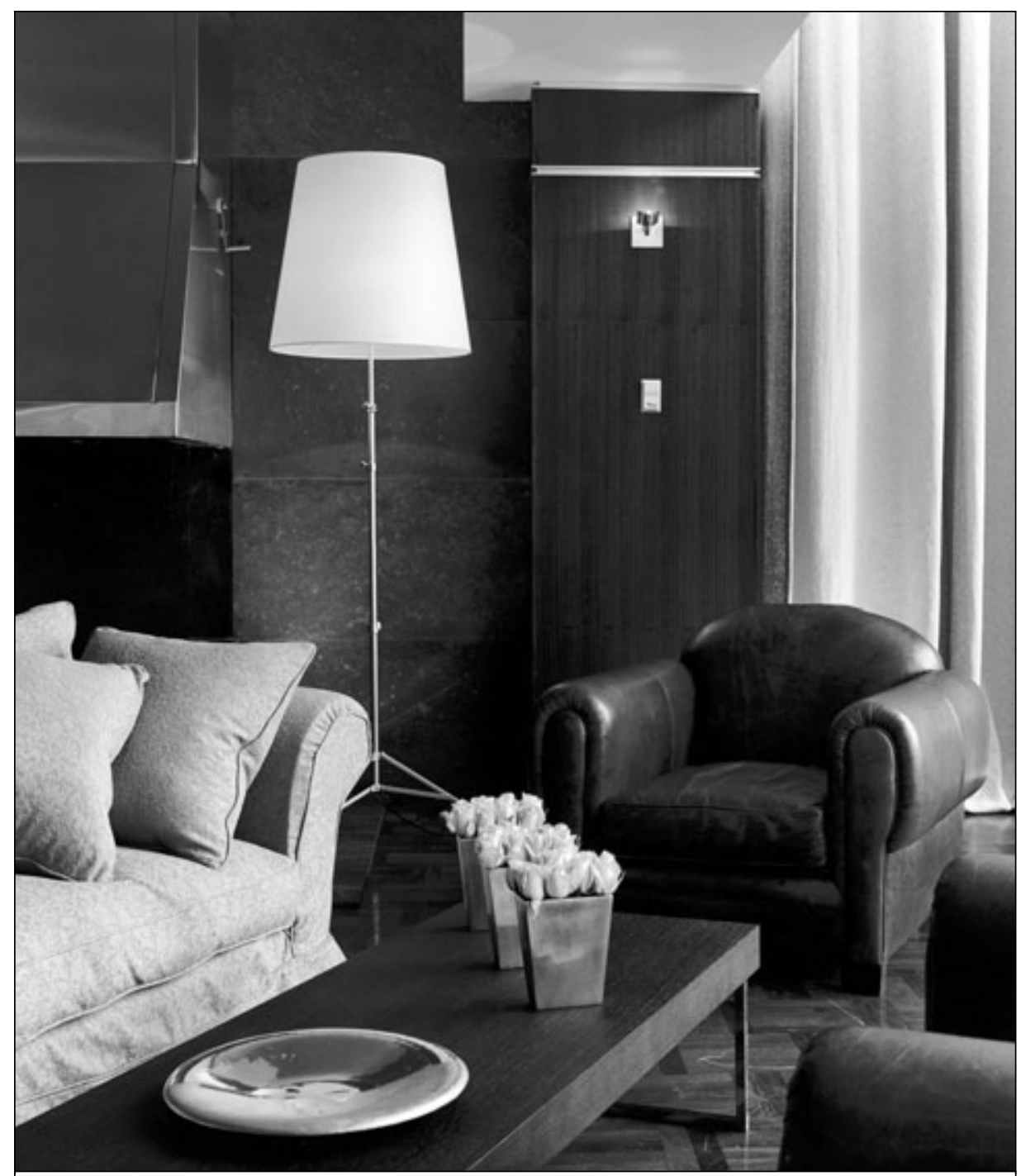

Suite à l'Hôtel Le Germain.

(c) Hôtel le Germain/Tourisme Montréa

\section{Conclusion}

L'évolution continue du mode de vie des populations, ainsi que le nouveau climat social forgé par la succession d'événements percutants survenus récemment, comme l'émergence de la menace réelle du terrorisme en sol nord-américain ou encore, plus près de nous, la vulnérabilité et l'inquiétude face à la résurgence de crises, comme celles causées par le syndrome respiratoire aigu grave (SRAS) ou l'encéphalopathie spongiforme bovine (maladie de la vache folle), redéfiniront sans doute la nature même de la demande touristique et, par le fait même, la demande hôtelière. Comme ces nouvelles réalités sont relativement récentes, il est cependant difficile d'en prévoir les effets à long terme. café préféré, son journal habituel ainsi qu'une foule de petites attentions personnalisées lui seront offerts dans sa chambre, sans même qu'il ait à demander quoi que ce soit, ne fait plus partie de la fiction. On se rend maintenant compte que « l'expérience de séjour » est véritablement devenue «le produit» de ce secteur et que «la chambre » ne joue plus qu'un rôle accessoire. Ce sont ceux qui auront vu venir ce changement qui marqueront l'avenir.

Gilles Larivière est président de Horwath Horizon Consultants, bureau de Montréal.

Jocelyn Jussaume agit à titre de conseiller chez Horwath Horizon Consultants, bureau de Montréal.

\section{Bibliographie}

Albazzaz, Alec et al. (2001), Lifestyle of the Rich and Almost Famous: The Boutique Hotel Phenomenon in the United States, High Tech Entrepreneurship and Strategy Group Project, 50 p.

Brock, Gary et al. (2002), The History of Lodging : The Hotel in America, p. 17 et 21-32.

Larivière, Gilles (2004), La nouvelle hôtellerie, Présentation à la Chaire de tourisme de l'Université du Québec à Montréal, Montréal.

Levy-Bonvin, Jacques (2003), « Hotels-A Brief History », Hospitality Net Article, décembre, p. 1-8, [www.hospitalitynet.org].

Nielsen, David, The Future of Hotels, [www.brunching.com/newhotels.html].

Pence, Paul, The Future of Hotel Stays, p. 1-4, [www.riroads.com/archive/futurehotel.htm]. à venir, il ne fait nul doute que les besoins du client seront plus que jamais au cœur des stratégies marketing des entreprises hôtelières, alors que les outils technologiques actuels permettent maintenant d'aller très loin en matière de détermination du profil des utilisateurs de produits et services. Les hôteliers ont maintenant tous les outils pour prendre avantage de la connaissance de leurs clients et ce, sur une base individuelle, dans le but de leur offrir des produits et services qui rejoignent directement leurs préférences.

L'arrivée d'un client à l'hôtel où l'attend un valet qu'il ne connaît pas et qui, pourtant, s'adressera à lui par son nom, et où son
Rutes, Walter, et Richard Penner (1985), Hotel Planning and Design, p. 30-36.

Valhouli, Christina (2004), «Hotels of the Future», Forbes, juin.

[www.msnbc.msn.com/id/5077355].

[www.nationmaster.com/encyclopedia/Timeline -of-transportation-technology].

[www.fairmont.com].

[www.whotels.com].

[www.ianschragerhotels.com].

[www.kimptonhotels.com]. 